\title{
LOCALIZATION FROM CONDUCTANCE IN FEW-CHANNEL DISORDERED WIRES
}

\author{
J.Heinrichs \\ Institut de physique, B5, Université de Liège, Sart Tilman, B-4000 Liège, Belgium
}

\begin{abstract}
We study localization in two- and three channel quasi-1D systems using multichain tight-binding Anderson models with nearest-neighbour interchain hopping. In the three chain case we discuss both the case of free- and that of periodic boundary conditions between the chains. The finite disordered wires are connected to ideal leads and the localization length is defined from the Landauer conductance in terms of the transmission coefficients matrix. The transmission- and reflection amplitudes in properly defined quantum channels are obtained from $S$-matrices constructed from transfer matrices in Bloch wave bases for the various quasi-1D systems. Our exact analytic expressions for localization lengths for weak disorder reduce to the Thouless expression for 1D systems in the limit of vanishing interchain hopping. For weak interchain hopping the localization length decreases with respect to the $1 \mathrm{D}$ value in all three cases. In the three-channel cases it increases with interchain hopping over restricted domains of large hopping. 72.10.-d, 73.70.+m, 73.23.-b - e-mail: J.Heinrichs@ulg.ac.be
\end{abstract}




\section{INTRODUCTION}

A wire is a topologically one-dimensional system whose width of the order of the square root of the cross-sectional area $A$ is much smaller than its length $L$ i.e. $\sqrt{A}<<L$. In a thin wire the motion of electrons in the transverse direction is quantized. The corresponding

transverse eigenstates below the Fermi level define a finite number, $N \propto \sqrt{\frac{2 A}{\lambda_{F}}}\left(\right.$ with $\lambda_{F}$, the Fermi wavelength), of quantum channels for transmission of electrons across the wire. The starting point of the present work is the Landauer two-probe conductance formula [1]:2]

$$
g=\frac{2 e^{2}}{h} \operatorname{Tr}\left(\hat{t}^{+}\right)
$$

which describes current transport in a disordered wire. Here $\hat{t}$ is the so-called transmission matrix of the $N$-channel system:

$$
\hat{t}=\left(\begin{array}{cccc}
t_{11} & t_{12} & \ldots & t_{1 N} \\
t_{21} & \ldots & \ldots & \ldots \\
\ldots & \ldots & \ldots & \\
t_{N 1} & t_{N 2} & \ldots & t_{N N}
\end{array}\right)
$$

An incoming wave from channel $j$ of an ideal lead at one end of the disordered wire (of length $L$ ) has a coefficient $\left|t_{i j}\right|^{2}$ for transmission into channel $i$ of the lead at the other end.

The correctness of the description of the conductance in terms of transmission channels has received striking experimental confirmation [3] in the special case of perfectly transmitting channels, where (11) reduces to

$$
g=\frac{2 e^{2}}{h} N
$$

These studies relate to quantum point contacts in the form of narrow conducting twodimensional strips whose width, hence the number of discrete transmitting channels, may 
be varied by varying externally applied gate voltages. Conductance steps corresponding to increasing values of $N$ in (3) are clearly observed [3] . A further interesting feature of these experiments is that they offer the possibility of realizing physically not only purely one-dimensional systems $(N=1)$ but also few-channel systems $(N=2,3, \ldots)$ such as those studied below. We recall that recent discussions of many-channel mesoscopic systems have been essentially restricted to the case $N>>1$ [4] i.e. real (metallic) wires whose widths are much larger than the Fermi wavelength.

The behaviour of the conductance in a disordered wire depends strongly on its length relative to the localization length $L_{c}$. In an infinitely long disordered wire of a given crosssection all eigenstates are expected to be localized just like in the truly one-dimensional case. Thouless has indeed shown [5, 6] that for $L>L_{c}$, where

$$
L_{c} \sim N \ell
$$

$\ell$ being the mean free path, the conductance should fall off exponentially as $e^{-L / L_{c}}$, which is a clear manifestation of localization. On the other hand, one the scales of lengths in the domain

$$
\ell<L<L_{c}=N \ell
$$

the eigenstates appear as being delocalized. In fact, it was shown later [7] that for lengths in the range (5) there remains a number $N_{\text {eff }} \sim \frac{N \ell}{L}$ of independent ballistic channels leading to metallic Ohm's law behaviour for the conductance (11). Such a diffusive quasi-metallic domain does not exist for truly one-dimensional systems where $L_{c} \equiv L_{1 c} \sim \ell$. Dorokhov 8 has developed a detailed scaling analysis [9] of localization in a multichannel wire, in which he calculates $L_{c}$ in terms of a phenomenological mean free path entering as input via Ohm's law at short scales. His final result, which is valid for weak disorder, is 


$$
L_{c}=(N+1) \ell
$$

which coincides with (4) for $N>>1$ and suggests that $L_{c}$ is not directly proportional to the number of channels for few channel systems. In particular, for one-dimensional systems it yields

$$
L_{1 c}=2 \ell
$$

We recall that (7) coincides with the result obtained long ago by Thouless [10] from kinetic transport theory. The same result follows also from the quantum series composition law for conductances $g=|t|^{2}$ for $1 \mathrm{D}$ conductors, assuming Ohm's law to be valid at short length scales [11]. On the other hand, we note that for two-channel systems (6) yields $L_{c} \equiv L_{2 c}=3 \ell$ which is comparable to the result $L_{2 c}=2 \ell\left(1-\frac{1}{\pi}\right)^{-1}$ obtained by Dorokhov 12 in a different scaling treatment.

Shortly before the development of the scaling theory of localization for 1D conductors [9], Thouless derived his well-known analytic expression for the quantum localization length in a tight-binding linear chain for weak disorder [13]. The purpose of the present work is to derive similar exact microscopic expressions for the localization length in quasi-one-dimensional few channel systems, specifically for two- and three chain systems for weak disorder. This study is of interest in several respects. For example, via the dependence of the localization length on the energy across the energy bands of the pure systems, it provides a first principles quantum proof of the fact that all states in the quasi-one-dimensional systems are localized. It is also relevant for experimental situations e.g. for discussing the quasi-metallic domain in few channel systems which are encountered in semiconducting microstructures [14] or may be fabricated artificially, as in the quantum point contact system discussed above.

In II.a we define the tight-binding two- and three chain systems for modelling few channel wires. In II.b we describe our methodology for studying the localization length. It 
consists in constructing successively transfer- and transmission-reflection matrices for the tight-binding systems by generalizing well-known methods for $1 \mathrm{D}$ systems. It further relies on general results concerning the existence and the properties of a Lyapunov exponent (inverse localization length) describing the asymptotic exponential decay of the conductance (11) in mutichannel systems [15]. In Sect. III we present the details of our calculations leading to the final analytic expressions for the transfer and scattering matrices for the twoand three channel wires for weak disorder. For the case $N=3$, we obtain different results for open- and for periodic boundary conditions which correspond to packing the chains on a plane and on a cylindrical surface, respectively. The final analytic expressions for the averaged transmission- and reflection coefficients and for localization lengths are discussed in Sect. IV. For clarity's sake some details of these calculations are relegated to an appendix.

\section{FEW-CHANNEL WIRES AND LOCALIZATION}

\section{A. Anderson models in channel bases}

We describe two $(N=2)$ - and three $(N=3)$-channel wires by Anderson models for two-

and three coupled chain systems, respectively. The two chain Anderson model consists of parallel linear chains of $N_{L}$ disordered sites each (of spacing $a=1$ and length $L=N_{L} a$ ) connected at both ends to semi-infinite ideal (non-disordered) leads. It is defined by the tight-binding Schrödinger equation which we write in the matrix form

$$
\left(\begin{array}{c}
\varphi_{n+1}^{1}+\varphi_{n-1}^{1} \\
\varphi_{n+1}^{2}+\varphi_{n-1}^{2}
\end{array}\right)=\left(\begin{array}{cc}
E-\varepsilon_{1 n} & -h \\
-h & E-\varepsilon_{2 n}
\end{array}\right)\left(\begin{array}{c}
\varphi_{n}^{1} \\
\varphi_{n}^{2}
\end{array}\right)
$$

where the $\varphi_{m}^{i}$ denote the wave-function amplitudes at sites $m$ on the chain $i, h$ is a constant matrix element for an electron to hop transversally between a site $n$ on chain 1 and its nearest-neighbour site $n$ on chain 2 . The site energies $\varepsilon_{i m}$ are random variables associated with the sites $1 \leq m \leq N_{L}$ of the disordered chain $i$, and $\varepsilon_{i m}=0$ on the semi-infinite ideal 
chains defined by the sites $m>N_{L}$ and $m<1$, respectively. The above energies, including $E$, are measured in units of the constant hopping rate along the individual chains.

The coupled three chain $(N=3)$ Anderson model is defined in a similar way by a set of tight-binding Schrödinger equations, whose actual form depends, however, on interchain boundary conditions. For free boundary conditions, which correspond to arranging the parallel equidistant chains on a plane, the tight-binding equations are

$$
\left(\begin{array}{c}
\varphi_{n+1}^{1}+\varphi_{n-1}^{1} \\
\varphi_{n+1}^{2}+\varphi_{n-1}^{2} \\
\varphi_{n+1}^{3}+\varphi_{n-1}^{3}
\end{array}\right)=\left(\begin{array}{ccc}
E-\varepsilon_{1 n} & -h & 0 \\
-h & E-\varepsilon_{2 n} & -h \\
0 & -h & E-\varepsilon_{3 n}
\end{array}\right)\left(\begin{array}{l}
\varphi_{n}^{1} \\
\varphi_{n}^{2} \\
\varphi_{n}^{3}
\end{array}\right)
$$

with the sites in the disordered sections of length $L=N_{L} a$ and in the semi-infinite ideal chain sections labelled in the same way as in the two chain case. On the other hand, in the case of periodic boundary conditions which correspond to equidistant linear chains on a cylindrical surface the Schrödinger equation is

$$
\left(\begin{array}{c}
\varphi_{n+1}^{1}+\varphi_{n-1}^{1} \\
\varphi_{n+1}^{2}+\varphi_{n-1}^{2} \\
\varphi_{n+1}^{3}+\varphi_{n-1}^{3}
\end{array}\right)=\left(\begin{array}{ccc}
E-\varepsilon_{1 n} & -h & -h \\
-h & E-\varepsilon_{2 n} & -h \\
-h & -h & E-\varepsilon_{3 n}
\end{array}\right)\left(\begin{array}{c}
\varphi_{n}^{1} \\
\varphi_{n}^{2} \\
\varphi_{n}^{3}
\end{array}\right)
$$

As discussed in Sect. I, a quasi-one-dimensional wire is described by a collection of independent channels for wave transmission. Microscopic models for two- and three channel wires are obtained from the systems of tight-binding equations (8-10) by diagonalizing the interchain coupling terms in the equations describing the ideal leads. This indeed leads to independent quantum channels for the leads defined by new amplitudes bases

$$
\left(\begin{array}{c}
\vdots \\
\psi_{n}^{i} \\
\vdots
\end{array}\right)=\widehat{U}^{-1}\left(\begin{array}{c}
\vdots \\
\varphi_{n}^{i} \\
\vdots
\end{array}\right)
$$


in which the non-random parts of the matrices on the r.h.s. of (8-10) are diagonal. For the multichain system above we obtain, respectively.

$$
\begin{aligned}
& \left(\begin{array}{l}
\psi_{n}^{1} \\
\psi_{n}^{2}
\end{array}\right)=\widehat{U}_{0}\left(\begin{array}{l}
\varphi_{n}^{1} \\
\varphi_{n}^{2}
\end{array}\right) \quad, \quad \widehat{U}_{0}=\frac{1}{\sqrt{2}}\left(\begin{array}{cc}
1 & 1 \\
1 & -1
\end{array}\right), \\
& \widehat{U}_{0}\left(\begin{array}{cc}
E & -h \\
-h & E
\end{array}\right) \widehat{U}_{0}=\left(\begin{array}{cc}
E-h & 0 \\
0 & E+h
\end{array}\right) \text {, } \\
& \left(\begin{array}{l}
\psi_{n}^{1} \\
\psi_{n}^{2} \\
\psi_{n}^{3}
\end{array}\right)=\widehat{U}^{\prime}\left(\begin{array}{l}
\varphi_{n}^{1} \\
\varphi_{n}^{2} \\
\varphi_{n}^{3}
\end{array}\right) \quad, \quad \widehat{U}^{\prime}=\frac{1}{2}\left(\begin{array}{ccc}
1 & \sqrt{2} & 1 \\
\sqrt{2} & 0 & -\sqrt{2} \\
1 & -\sqrt{2} & 1
\end{array}\right) \\
& \widehat{U}^{\prime}\left(\begin{array}{ccc}
E & -h & 0 \\
-h & E & -h \\
0 & -h & E
\end{array}\right) \widehat{U}^{\prime}=\left(\begin{array}{ccc}
E-\sqrt{2} h & 0 & 0 \\
0 & E & 0 \\
0 & 0 & E+\sqrt{2} h
\end{array}\right), \\
& \left(\begin{array}{c}
\psi_{n}^{1} \\
\psi_{n}^{2} \\
\psi_{n}^{3}
\end{array}\right)=(\widehat{U} \prime \prime)^{-1}\left(\begin{array}{c}
\varphi_{n}^{1} \\
\varphi_{n}^{2} \\
\varphi_{n}^{3}
\end{array}\right) \quad, \quad \widehat{U} " \prime=\left(\begin{array}{ccc}
1 & 1 & 0 \\
1 & 0 & 1 \\
1 & -1 & -1
\end{array}\right), \\
& \left(\widehat{U}^{\prime \prime}\right)^{-1}\left(\begin{array}{ccc}
E & -h & -h \\
-h & E & -h \\
-h & -h & E
\end{array}\right) \widehat{U} "=\left(\begin{array}{ccc}
E-2 h & 0 & 0 \\
0 & E+h & 0 \\
0 & 0 & E+h
\end{array}\right) .
\end{aligned}
$$

Note that $\widehat{U}_{0}$ and $\widehat{U}^{\prime}$ are unitary, while $\widehat{U}$ ' is not.

Finally, in the channel bases defined by (8a-10a) the tight-binding equations (810) read, respectively,

$$
\left(\begin{array}{c}
\psi_{n+1}^{1}+\psi_{n-1}^{1} \\
\psi_{n+1}^{2}+\psi_{n-1}^{2}
\end{array}\right)=\left(\begin{array}{cc}
E-h-\frac{1}{2}\left(\varepsilon_{1 n}+\varepsilon_{2 n}\right) & \frac{1}{2}\left(\varepsilon_{2 n}-\varepsilon_{1 n}\right) \\
\frac{1}{2}\left(\varepsilon_{2 n}-\varepsilon_{1 n}\right) & E+h-\frac{1}{2}\left(\varepsilon_{1 n}+\varepsilon_{2 n}\right)
\end{array}\right)\left(\begin{array}{l}
\psi_{n}^{1} \\
\psi_{n}^{2}
\end{array}\right),
$$




$$
\begin{aligned}
& \left(\begin{array}{c}
\psi_{n+1}^{1}+\psi_{n-1}^{1} \\
\psi_{n+1}^{2}+\psi_{n-1}^{2} \\
\psi_{n+1}^{3}+\psi_{n-1}^{3}
\end{array}\right)= \\
& \left(\begin{array}{ccc}
E-\sqrt{2} h-\frac{1}{4}\left(\varepsilon_{1 n}+2 \varepsilon_{2 n}+\varepsilon_{3 n}\right) & \frac{\sqrt{2}}{4}\left(\varepsilon_{3 n}-\varepsilon_{1 n}\right) & -\frac{1}{4}\left(\varepsilon_{1 n}-2 \varepsilon_{2 n}+\varepsilon_{3 n}\right) \\
\frac{\sqrt{2}}{4}\left(\varepsilon_{3 n}-\varepsilon_{1 n}\right) & E-\frac{1}{2}\left(\varepsilon_{1 n}+\varepsilon_{3 n}\right) & \frac{\sqrt{2}}{4}\left(\varepsilon_{3 n}-\varepsilon_{1 n}\right) \\
-\frac{1}{4}\left(\varepsilon_{1 n}-2 \varepsilon_{2 n}+\varepsilon_{3 n}\right) & \frac{\sqrt{2}}{4}\left(\varepsilon_{3 n}-\varepsilon_{1 n}\right) & E+\sqrt{2} h-\frac{1}{4}\left(\varepsilon_{1 n}+2 \varepsilon_{2 n}+\varepsilon_{3 n}\right)
\end{array}\right)\left(\begin{array}{l}
\psi_{n}^{1} \\
\psi_{n}^{2} \\
\psi_{n}^{3}
\end{array}\right)
\end{aligned}
$$

$$
\begin{aligned}
& \left(\begin{array}{c}
\psi_{n+1}^{1}+\psi_{n-1}^{1} \\
\psi_{n+1}^{2}+\psi_{n-1}^{2} \\
\psi_{n+1}^{3}+\psi_{n-1}^{3}
\end{array}\right)= \\
& \left(\begin{array}{ccc}
E-2 h-\frac{1}{3}\left(\varepsilon_{1 n}+\varepsilon_{2 n}+\varepsilon_{3 n}\right) & -\frac{1}{3}\left(\varepsilon_{1 n}-\varepsilon_{3 n}\right) & -\frac{1}{3}\left(\varepsilon_{2 n}-\varepsilon_{3 n}\right) \\
-\frac{1}{3}\left(2 \varepsilon_{1 n}-2 \varepsilon_{2 n}-\varepsilon_{3 n}\right) & E+h-\frac{1}{3}\left(\varepsilon_{1 n}+\varepsilon_{3 n}\right) & \frac{1}{3}\left(\varepsilon_{2 n}-\varepsilon_{3 n}\right) \\
\frac{1}{3}\left(\varepsilon_{1 n}-2 \varepsilon_{2 n}+\varepsilon_{3 n}\right) & \frac{1}{3}\left(\varepsilon_{1 n}-\varepsilon_{3 n}\right) & E+h-\frac{1}{3}\left(2 \varepsilon_{2 n}+\varepsilon_{3 n}\right)
\end{array}\right)\left(\begin{array}{l}
\psi_{n}^{1} \\
\psi_{n}^{2} \\
\psi_{n}^{3}
\end{array}\right)
\end{aligned}
$$

which constitute our starting point for deriving transmission- and reflection matrices of the disordered wires in Sect. III. It is seen that the similarity transformation of the disorder matrices by the $\widehat{U}$-matrices leads to interchannel coupling in the disordered sections, $1 \leq$ $N \leq N_{L}$

\section{B. Localization from conductance}

In Sect. IV we will calculate the localization length in the above multi-channel wire models from the rate of exponential decrease (Lyapunov exponent) of the conductance (1) for the large $L$ [15]. The transmission matrix in (2) will be found by constructing a transfer matrix which transforms propagating waves in the multichannel leads (defined by (8.a-10.a) on the left side of the disordered wire into corresponding propagating waves on the right side. The transfer matrix for the wire of length $L$ is expressed, as usual, as a product of $N_{L}$ 
transfer matrices for small sections enclosing only the $n$th site of each one of the channels. The calculation of the localization length rests on theorems of Oseledec [16] and of Tutubalin and Vister [17] on the properties of products of a large number of random matrices. Indeed, employing these properties Johnston anf Kunz [15] have shown that the Lyapunov exponent $\gamma$ exists for the conductance (11) and is a self-averaging quantity referred to as the inverse localization length. It is defined by the relation

$$
\gamma \equiv \frac{1}{L_{c}}=-\lim _{N_{L} \rightarrow \infty} \frac{1}{2 N_{L}}\langle\ln g\rangle
$$

where $\langle\ldots\rangle$ denotes averaging over the disorder (i.e. the random site energies in the Anderson model) [18]. It follows that the asymptotic distribution of the conductance is log-normal.

\section{DETAILED ANALYSIS}

As indicated above the transmission matrices of the form (2) for the quasi 1D-disordered systems above will be obtained from transfer matrices for wavefunction amplitudes defined from equations (8.b-10.b), respectively. The construction of these transfer matrices proceeds in two steps. First, we will define transfer matrices for thin slices enclosing only one site $n$ of each chain in a disordered wire, in a Bloch plane wave basis. Next the transfer matrix of a whole wire of length $L=N_{L} a$ will be obtained as a product of the transfer matrices for the $N_{L}$ individual slices composing the wire. We will express it analytically to lowest order in the effect of a weak disorder. Finally we obtain the form of the tight-binding equations describing the transfer of Bloch wave amplitudes across a whole disordered wire of length $L$, which we then cast in the form of scattering equations in order to identify microscopic scattering matrices of the form (2), in terms of elements of the transfer matrices. We recall that the transfer matrix method is well-known in the study of one-dimensional disordered systems [19 21]. Here we generalize and adapt it in the case of few channel quasi-1D systems. 


\section{A. Transfer matrices}

Transfer matrices $\tilde{X}_{0 n}, \tilde{X}_{n}^{\prime}$ and $\tilde{X}_{n}$ " for thin slices including a single site $n$ per chain of the quasi-1D systems described by (8.b-10.b) are defined by rewrtiting these equations respectively in the forms

$$
\begin{gathered}
\left(\begin{array}{c}
\psi_{n+1}^{1} \\
\psi_{n}^{1} \\
\psi_{n+1}^{2} \\
\psi_{n}^{2}
\end{array}\right)=\tilde{X}_{0 n}\left(\begin{array}{c}
\psi_{n}^{1} \\
\psi_{n-1}^{1} \\
\psi_{n}^{2} \\
\psi_{n-1}^{2}
\end{array}\right), \\
\left(\begin{array}{c}
\psi_{n+1}^{1} \\
\psi_{n}^{1} \\
\psi_{n+1}^{2} \\
\psi_{n}^{2} \\
\psi_{n+1}^{3} \\
\psi_{n}^{3}
\end{array}\right)=\tilde{Y}_{n}\left(\begin{array}{c}
\psi_{n}^{1} \\
\psi_{n-1}^{1} \\
\psi_{n}^{2} \\
\psi_{n-1}^{2} \\
\psi_{n}^{3} \\
\psi_{n-1}^{3}
\end{array}\right), \tilde{Y}_{n} \equiv \tilde{X}_{n}^{\prime}, \tilde{X}_{n},
\end{gathered}
$$

where

$$
\tilde{X}_{0 n}=\left(\begin{array}{cccc}
E-h-\mu_{n} & -1 & \nu_{n} & 0 \\
1 & 0 & 0 & 0 \\
\nu_{n} & 0 & E+h-\mu_{n}-1 \\
0 & 0 & 1 & 0
\end{array}\right),
$$

$$
\mu_{n}=\frac{1}{2}\left(\varepsilon_{1 n}+\varepsilon_{2 n}\right), \nu_{n}=\frac{1}{2}\left(\varepsilon_{2 n}-\varepsilon_{1 n}\right) \quad,
$$




$$
\begin{aligned}
& \tilde{X}_{n}^{\prime}=\left(\begin{array}{cccccc}
E-\sqrt{2} h-\mu_{n}^{\prime} & -1 & \nu_{n}^{\prime} & 0 & \tau_{n}^{\prime} & 0 \\
1 & 0 & 0 & 0 & 0 & 0 \\
\nu_{n}^{\prime} & 0 & E-\eta_{n}^{\prime}-1 & \nu_{n}^{\prime} & 0 \\
0 & 0 & 1 & 0 & 0 & 0 \\
\tau_{n}^{\prime} & 0 & \nu_{n}^{\prime} & 0 & E+\sqrt{2} h-\mu_{n}^{\prime}-1 \\
0 & 0 & 0 & 0 & 1 & 0
\end{array}\right), \\
& \mu_{n}^{\prime}=\frac{1}{4}\left(\varepsilon_{1 n}+2 \varepsilon_{2 n}+\varepsilon_{3 n}\right), \nu_{n}^{\prime}=\frac{\sqrt{2}}{4}\left(\varepsilon_{3 n}-\varepsilon_{1 n}\right) \\
& \tau_{n}^{\prime}=-\frac{1}{4}\left(\varepsilon_{1 n}-2 \varepsilon_{2 n}+\varepsilon_{3 n}\right), \eta_{n}^{\prime}=\frac{1}{2}\left(\varepsilon_{3 n}+\varepsilon_{1 n}\right) \text {, } \\
& \tilde{X}_{n} "=\left(\begin{array}{cccccc}
E-2 h-\mu_{n} " & -1 & \nu_{n} " & 0 & \tau_{n} " & 0 \\
1 & 0 & 0 & 0 & 0 & 0 \\
\alpha_{n} " & 0 & E+h-\eta_{n} " & -1 & -\tau_{n} " & 0 \\
0 & 0 & 1 & 0 & 0 & 0 \\
\beta_{n} " & 0 & -\nu_{n} " & 0 & E+h-\theta_{n} " & -1 \\
0 & 0 & 0 & 0 & 1 & 0
\end{array}\right), \\
& \mu_{n} "=\frac{1}{3}\left(\varepsilon_{1 n}+\varepsilon_{2 n}+\varepsilon_{3 n}\right), \eta_{n} "=\frac{1}{3}\left(2 \varepsilon_{1 n}+\varepsilon_{3 n}\right), \theta_{n} "=\frac{1}{3}\left(2 \varepsilon_{2 n}+\varepsilon_{3 n}\right), \\
& \nu_{n} "=-\frac{1}{3}\left(\varepsilon_{1 n}-\varepsilon_{3 n}\right), \tau_{n} "=-\frac{1}{3}\left(\varepsilon_{2 n}-\varepsilon_{3 n}\right) \quad, \\
& \alpha_{n} "=-\frac{1}{3}\left(2 \varepsilon_{1 n}-\varepsilon_{2 n}-\varepsilon_{3 n}\right), \beta_{n} "=\frac{1}{3}\left(\varepsilon_{1 n}-2 \varepsilon_{2 n}+\varepsilon_{3 n}\right) \text {. }
\end{aligned}
$$

The study of disordered wires in terms of reflection and transmission properties of plane waves requires determination of plane wave bases in which the transfer matrices for slices $n$ in the leads are diagonal. Such bases are provided by the Bloch wave solutions for the leads which are defined by 


$$
\begin{gathered}
\tilde{X}_{00}\left(\begin{array}{c}
\psi_{n, \pm}^{1} \\
\psi_{n-1, \pm}^{1} \\
\psi_{n, \pm}^{2} \\
\psi_{n-1, \pm}^{2}
\end{array}\right)=\left(\begin{array}{c}
e^{ \pm i k_{1}} \psi_{n, \pm}^{1} \\
e^{ \pm i k_{1}} \psi_{n-1, \pm}^{1} \\
e^{ \pm i k_{2}} \psi_{n, \pm}^{2} \\
e^{ \pm i k_{2}} \psi_{n-1, \pm}^{2}
\end{array}\right), \\
\tilde{Y}_{0}\left(\begin{array}{c}
\psi_{n, \pm}^{1} \\
\psi_{n-1, \pm}^{1} \\
\psi_{n, \pm}^{2} \\
\psi_{n-1, \pm}^{2} \\
\psi_{n, \pm}^{3} \\
\psi_{n-1, \pm}^{3}
\end{array}\right)=\left(\begin{array}{c}
e^{ \pm i k_{1}} \psi_{n, \pm}^{1} \\
e^{ \pm i k_{1}} \psi_{n-1, \pm}^{1} \\
e^{ \pm i k_{2}} \psi_{n, \pm}^{2} \\
e^{ \pm i k_{2}} \psi_{n-1, \pm}^{2} \\
e^{ \pm i k_{3}} \psi_{n, \pm}^{3} \\
e^{ \pm i k_{3}} \psi_{n-1, \pm}^{3}
\end{array}\right), \tilde{Y}_{0} \equiv \tilde{X}_{0}^{\prime}, \tilde{X}_{0} "
\end{gathered}
$$

where $\tilde{X}_{00}, \tilde{X}_{0}^{\prime}$ and $\tilde{X}_{0}$ " denote the transfer matrices for the leads given by (14-16) with $\varepsilon_{1 n}=\varepsilon_{2 n}=\varepsilon_{3 n}=0$, respectively. The wavenumbers, $k_{i}$, are defined in terms of the energy $E$ by the eigenvalues of Eqs. (17) and (18), respectively. By solving for the eigenvalues we get successively

$$
\begin{aligned}
& 2 \cos k_{1}=E-h, \\
& 2 \cos k_{2}=E+h,
\end{aligned}
$$

for the two-channel system,

$$
\begin{aligned}
& 2 \cos k_{1}=E-\sqrt{2} h, \\
& 2 \cos k_{2}=E, \\
& 2 \cos k_{3}=E+\sqrt{2} h,
\end{aligned}
$$

for the three-channel system with free boundary conditions, whose leads are described by $\tilde{X}_{0}^{\prime}$, and, finally,

$$
\begin{aligned}
& 2 \cos k_{1}=E-2 h, \\
& 2 \cos k_{2}=2 \cos k_{3}=E+h,
\end{aligned}
$$


for the three-channel model with periodic lateral boundary conditions. The eigenfunctions in the leads at energy $E$ obtained from $12-13)$ and (17-18) are of the form

$$
\psi_{n, \pm}^{j} \sim e^{ \pm i n k_{j}}
$$

where we choose the wavenumbers $k_{j}, j=1,2,3$ to be positive, $0 \leq k_{j} \leq \pi$, so that these functions correspond to plane waves travelling from left to right and from right to left, respectively.

The transfer matrices for single site slices in Eqs (12-13) for the leads (i.e. for $\varepsilon_{i n}=0$, $n<1$ or $n>N)$ are diagonalized in the bases of the Bloch plane wave states (19). The diagonalization matrices which are formed by the eigenvectors of (17-18), are of the form

$$
\tilde{V}_{0}=\left(\begin{array}{cc}
\widehat{A}_{1} & \widehat{O} \\
\widehat{O} & \widehat{A}_{2}
\end{array}\right) \quad, \quad \widehat{A}_{j}=\frac{1}{\sqrt{2 i \sin k_{j}}}\left(\begin{array}{cc}
e^{i k_{j}} & e^{-i k_{j}} \\
1 & 1
\end{array}\right) \text {, }
$$

for the two-channel quasi-1D model, and

$$
\tilde{V}=\left(\begin{array}{ccc}
\widehat{A_{1}} & \widehat{O} & \widehat{O} \\
\widehat{O} & \widehat{A_{2}} & \widehat{O} \\
\widehat{O} & \widehat{O} & \widehat{A}_{3}
\end{array}\right)
$$

with $\widehat{A}_{j}$ defined as in (20), for the three-channel models. The wavenumbers $k_{j}$ defined by (17a) for the $N=2$ case and by (18.a) and (18b) for the $N=3$ case with free- and periodic boundary conditions, respectively. After finding the inverses of $\widehat{V}_{0}$ and $\widehat{V}$ and performing the simularity transformations of $\tilde{X}_{0 n}, \tilde{X}_{n}^{\prime}$ and $\tilde{X}_{n}$ " by $\widehat{V}_{0}$ and $\widehat{V}$, respectively, we obtain the desired transfer matrices in the Bloch wave representation of the disordered wires. In the two-channel case we find 


$$
\begin{aligned}
\widehat{X}_{0 n} \equiv & \widehat{V}_{0}^{-1} \tilde{X}_{0 n} \widehat{V}_{0} \\
= & \left(\begin{array}{cccc}
e^{i k_{1}}\left(1+i a_{1 n}\right) & i e^{-i k_{1}} a_{1 n} & -i e^{i k_{2}} b_{n} & -i e^{i k_{2}} b_{n} \\
-i e^{i k_{1}} a_{1 n} & e^{-i k_{1}}\left(1-i a_{1 n}\right) & i e^{i k_{2}} b_{n} & i e^{-i k_{2}} b_{n} \\
-i e^{i k_{1}} b_{n} & -i e^{-i k_{1}} b_{n} & e^{i k_{2}}\left(1+i a_{2 n}\right) & i e^{-i k_{2}} a_{2 n} \\
i e^{i k_{1}} b_{n} & i e^{-i k_{1}} b_{n} & -i e^{i k_{2}} b_{n} & e^{-i k_{2}}\left(1-i a_{2 n}\right)
\end{array}\right),
\end{aligned}
$$

where

$$
\begin{aligned}
a_{1 n} & =\frac{\varepsilon_{1 n}+\varepsilon_{2 n}}{4 \sin k_{1}}, a_{2 n} \frac{\varepsilon_{1 n}+\varepsilon_{2 n}}{4 \sin k_{2}}, \\
b_{n} & =\frac{\varepsilon_{2 n}-\varepsilon_{1 n}}{4 \sqrt{\sin k_{1} \sin k_{2}}}
\end{aligned}
$$

are real quantities and $k_{1}, k_{2}$ are defined by (17a). For the three-channel systems we write the final transfer matrices $\widehat{X}_{n}^{\prime}$ and $\widehat{X}^{\prime}{ }_{n}$, in terms of a generic matrix

$$
\begin{aligned}
& \widehat{Z}_{n}= \\
& \left(\begin{array}{cccccc}
e^{i k_{1}}\left(1+i a_{1 n}\right) & i e^{-i k_{1}} a_{1 n} & i e^{i k_{2}} c_{n} & i e^{-i k_{2}} c_{n} & i e^{i k_{3}} g_{n} & i e^{-i k_{3}} g_{n} \\
-i e^{i k_{1}} a_{1 n} & e^{-i k_{1}}\left(1-i a_{1 n}\right) & -i e^{i k_{2}} c_{n} & -i e^{-i k_{2}} c_{n} & -i e^{i k_{3}} g_{n} & -i e^{-i k_{3}} g_{n} \\
i e^{i k_{1}} f_{n} & i e^{-i k_{1}} f_{n} & e^{i k_{2}}\left(1+i b_{2 n}\right) & i e^{-i k_{2}} b_{2 n} & i e^{i k_{3}} d_{n} & i e^{-i k_{3}} d_{n} \\
-i e^{i k_{1}} f_{n} & -i e^{-i k_{1}} f_{n} & -i e^{i k_{2}} b_{2 n} & e^{-i k_{2}}\left(1-i b_{2 n}\right) & -i e^{i k_{3}} d_{n} & -i e^{-i k_{3}} d_{n} \\
i e^{i k_{1}} p_{n} & i e^{-i k_{1}} p_{n} & i e^{i k_{2}} q_{n} & i e^{-i k_{2}} q_{n} & e^{i k_{3}}\left(1+i a_{3 n}\right) & i e^{-i k_{3}} a_{3 n} \\
-i e^{i k_{1}} p_{n} & i e^{-i k_{1}} p_{n} & -i e^{i k_{2}} q_{n} & -i e^{-i k_{2}} q_{n} & -i e^{i k_{3}} a_{3 n} & e^{-i k_{3}}\left(1-i a_{3 n}\right)
\end{array}\right)
\end{aligned}
$$

where $a_{1 n}, a_{3 n}, b_{2 n}, c_{n}, g_{n}, f_{n}, d_{n}, p_{n}$ and $q_{n}$ are real quantities. In the case of free boundary conditions we obtain $\widehat{X}_{n}^{\prime}=\widehat{V}^{-1} \tilde{X}_{n}^{\prime} \widehat{V} \equiv \widehat{Z}_{n}$ with

$$
\begin{gathered}
a_{1 n}=\frac{\varepsilon_{1 n}+2 \varepsilon_{2 n}+\varepsilon_{3 n}}{8 \sin k_{1}}, a_{3 n}=\frac{\varepsilon_{1 n}+2 \varepsilon_{2 n}+\varepsilon_{3 n}}{8 \sin k_{3}}, \\
b_{2 n}=\frac{\varepsilon_{1 n}+\varepsilon_{3 n}}{4 \sin k_{2}}, c_{n}=f_{n}=\frac{\sqrt{2}\left(\varepsilon_{1 n}-\varepsilon_{3 n}\right)}{8 \sqrt{\sin k_{1} \sin k_{2}}}, \\
d_{n}=q_{n}=\frac{\sqrt{2}\left(\varepsilon_{1 n}-\varepsilon_{3 n}\right)}{8 \sqrt{\sin k_{2} \sin k_{3}}}, g_{n}=p_{n}=\frac{\varepsilon_{1 n}-2 \varepsilon_{2 n}+\varepsilon_{3 n}}{8 \sqrt{\sin k_{1} \sin k_{3}}} .
\end{gathered}
$$

Here $k, k_{1}, k_{3}$ are defined by (18.a). On the other hand, for periodic boundary conditions we find $\widehat{X}_{n}{ }_{n}=\widehat{V}^{-1} \tilde{X} " \widehat{V} \equiv \widehat{Z}_{n}$ where 


$$
\begin{aligned}
a_{1 n} & =\frac{\varepsilon_{1 n}+\varepsilon_{2 n}+\varepsilon_{3 n}}{6 \sin k_{1}}, a_{3 n}=\frac{2 \varepsilon_{2 n}+\varepsilon_{3 n}}{6 \sin k_{2}}, \\
b_{2 n} & =\frac{2 \varepsilon_{1 n}+\varepsilon_{3 n}}{6 \sin k_{2}}, c_{n}=\frac{\varepsilon_{1 n}-\varepsilon_{3 n}}{6 \sqrt{\sin k_{1} \sin k_{2}}}, g_{n}=\frac{\varepsilon_{2 n}-\varepsilon_{3 n}}{6 \sqrt{\sin k_{1} \sin k_{2}}}, \\
d_{n} & =\frac{\varepsilon_{3 n}-\varepsilon_{2 n}}{6 \sin k_{2}}, f_{n}=\frac{2 \varepsilon_{1 n}-\varepsilon_{2 n}-\varepsilon_{3 n}}{6 \sqrt{\sin k_{1} \sin k_{2}}}, \\
p_{n} & =\frac{-\varepsilon_{1 n}+2 \varepsilon_{2 n}-\varepsilon_{3 n}}{6 \sqrt{\sin k_{1} \sin k_{2}}}, q_{n}=\frac{\varepsilon_{3 n}-\varepsilon_{1 n}}{6 \sin k_{2}},
\end{aligned}
$$

where $k_{1}$ and $k_{2}=k_{3}$ are now given by (18b). Note, in particular, the diagonalization of the transfer matrices for the leads in the plane wave bases shown in (22) 23$)$.

Finally, we determine the transfer matrices for the disordered wires of length $L=N_{L} a$ in terms of the transfer matrices of the individual thin slices $n$. As shown by iteration of the transfer equations (17 18) rewritten in the Bloch wave basis above, the matrix transferring an incoming wave at site $n=0$ just outside a disordered wire to the site $N_{L}+1$ just beyond its other end is given by a product of transfer matrices of the form

$$
\widehat{Y}_{L}=\prod_{n=1}^{N_{L}} \widehat{Y}_{n}
$$

where $\widehat{Y}_{n}$ and $\widehat{Y}_{L}$ stand for the three pairs of transfer matrices $\widehat{X}_{0 n}, \widehat{X}_{0 L}, \widehat{X}_{n}^{\prime}, \widehat{X}_{L}^{\prime}$ and $\widehat{X}_{n} ", \widehat{X}_{L} "$, respectively, which are associated with the wire models above [19].

We shall evaluate the transfer matrices of the disordered wires for weak disorder to linear order in the random site energies. On the other hand, for our explicit calculations of averages over the disorder below, we assume the site energies to be independent gaussian random variables with zero mean values, and correlation

$$
\left\langle\varepsilon_{i n} \varepsilon_{j m}\right\rangle=\varepsilon_{0}^{2} \delta_{i, j} \delta_{m, n}
$$

In this case the site energies corresponding to different slices $n$ in (26) are uncorrelated so that it is indeed sufficient to restrict the expansion of the latter expressions to first order in the site energies for determining averages to order $\varepsilon_{0}^{2}$. The transfer matrices $\widehat{Y}_{n}$ given by (22-23) are sums, 


$$
\widehat{Y}_{n}=\widehat{Y}^{(0)}+\widehat{Y}_{n}^{(1)}
$$

of a zeroth order diagonal matrix $\widehat{Y}^{(0)}$ independent of the site energies (transfer matrix of the leads) and a matrix which is linear in the energies $\varepsilon_{j n}$. By inserting (28) into (26) we obtain to first order

$$
\widehat{Y}_{L}=\left(\widehat{Y}^{(0)}\right)^{N_{L}}+\sum_{m=1}^{N_{L}}\left(\widehat{Y}^{(0)}\right)^{m-1} \widehat{Y}_{m}^{(1)}\left(\widehat{Y}^{(0)}\right)^{N_{L}-m}+\cdots
$$

Next we insert the slice matrices $\widehat{Y}^{(0)}$ and $\widehat{Y}_{m}^{(1)}$ from (22-23) for our various quasi-1D systems and obtain successively:

$$
\begin{aligned}
& \widehat{X}_{0 L}=\operatorname{diag}\left(e^{i k_{1} N_{L}}, e^{-i k_{1} N_{L}}, e^{i k_{2} N_{L}}, e^{-i k_{2} N_{L}}\right) \\
& +\sum_{m=1}^{N_{L}}\left(\begin{array}{cccc}
i a_{1 m} e^{i k_{1}} u_{1} & i a_{1 m} e^{-i k_{1}} s_{1}^{*} & -i b_{m} e^{i k_{2}} w_{21} & -i b_{1 m} e^{-i k_{2}} v_{21}^{*} \\
-i a_{1 m} e^{i k_{1}} s_{1} & -i a_{1 m} e^{-i k_{1}} u_{1}^{*} & i b_{m} e^{i k_{2}} v_{21} & i b_{1 m} e^{-i k_{2}} w_{21}^{*} \\
-i b_{m} e^{i k_{1}} w_{12} & -i b_{m} e^{-i k_{1}} v_{12}^{*} & i a_{2 m} e^{i k_{2}} u_{2} & i a_{2 m} e^{-i k_{2}} s_{2}^{*} \\
i b_{m} e^{i k_{1}} v_{12} & i b_{m} e^{-i k_{1}} w_{12}^{*} & -i a_{2 m} e^{i k_{2}} s_{2} & -i a_{2 m} e^{-i k_{2}} u_{2}^{*}
\end{array}\right),
\end{aligned}
$$

for the two-channel case. Here

$$
\begin{aligned}
s_{j} & =e^{i\left(N_{L}-2 m+1\right) k_{j}}, u_{j}=e^{i\left(N_{L}-1\right) k_{j}}, \\
v_{i j} & =e^{i\left(N_{L}-m\right) k_{i}-i(m-1) k_{j}}, w_{i j}=e^{i\left(N_{L}-m\right) k_{i}+i(m-1) k_{j}},
\end{aligned}
$$

where $k_{1}$ and $k_{2}$ are defined by (17a);

$$
\begin{aligned}
\widehat{X}_{L}^{\prime}= & \operatorname{diag}\left(e^{i k_{1} N_{L}}, e^{-i k_{1} N_{L}}, e^{i k_{2} N_{L}}, e^{-i k_{2} N_{L}}, e^{i k_{3} N_{L}}, e^{-i k_{3} N_{L}}\right)+\sum_{m=1}^{N_{L}} \\
& \left(\begin{array}{cccccc}
i a_{1 m} e^{i k_{1}} u_{1} & i a_{1 m} e^{-i k_{1}} s_{1}^{*} & i c_{m} e^{i k_{2}} w_{21} & i c_{m} e^{-i k_{2}} v_{21}^{*} & i g_{m} e^{i k_{3}} w_{31} & i g_{m} e^{-i k_{3}} v_{31}^{*} \\
-i a_{1 m} e^{i k_{1}} s_{1} & -i a_{1 m} e^{-i k_{1}} u_{1}^{*} & -i c_{m} e^{i k_{2}} v_{21} & -i c_{m} e^{-i k_{2}} w_{21}^{*} & -i g_{m} e^{i k_{3}} v_{31} & -i g_{m} e^{-i k_{3}} w_{31}^{*} \\
i f_{m} e^{i k_{1}} w_{12} & i f_{m} e^{-i k_{1}} v_{12}^{*} & i b_{2 m} e^{i k_{2}} u_{2} & i b_{2 m} e^{-i k_{2}} s_{2}^{*} & i d_{m} e^{i k_{3}} w_{32} & i d_{m} e^{-i k_{3}} v_{32}^{*} \\
-i f_{m} e^{i k_{1}} v_{12} & -i f_{m} e^{-i k_{1}} w_{12}^{*} & -i b_{2 m} e^{i k_{2}} s_{2} & -i b_{2 m} e^{-i k_{2}} u_{2}^{*} & -i d_{m} e^{i k_{3}} v_{32} & -i d_{m} e^{-i k_{3}} w_{32}^{*} \\
i p_{m} e^{i k_{1}} w_{13} & i p_{m} e^{-i k_{1}} v_{13}^{*} & i q_{m} e^{i k_{2}} w_{23} & i q_{m} e^{-i k_{2}} v_{23}^{*} & i a_{3 m} e^{i k_{3}} u_{3}^{*} & i a_{3 m} e^{-i k_{3}} s_{3}^{*} \\
-i p_{m} e^{i k_{1}} v_{13} & -i p_{m} e^{-i k_{1}} w_{13}^{*} & -i q_{m} e^{i k_{2}} w_{23} & -i q_{m} e^{-i k_{2}} w_{23}^{*} & -i a_{3 m} e^{i k_{3}} s_{3}^{*} & -i a_{3 m} e^{-i k_{3}} u_{3}^{*}
\end{array}\right),
\end{aligned}
$$


for the three-channel case with free boundary conditions where $k_{1}, k_{2}, k_{3}$ in the definitions (24) and (31) are given by (18.a); the transfer matrix $\widehat{X}_{L}$ " for the three-channel model with periodic boundary conditions is given by (32), using the definitions (25) of the slice parameters $a_{1 m}, a_{3 m}, b_{2 m}, c_{m}$ and the definition $(18 \mathrm{~b})$ of the wavenumbers $k_{1}, k_{2}, k_{3}$.

\section{B. Scattering matrices}

The scattering of plane waves (reflection and transmission) at and between the two ends of the random quasi-1D systems is governed by the $S$-matrix,

$$
\widehat{S}=\left(\begin{array}{cc}
\hat{r}^{-+} & \hat{t}^{--} \\
\hat{t}^{++} & \hat{r}^{+-}
\end{array}\right)
$$

where

$$
\hat{t}^{\mp \mp}=\left(\begin{array}{ccc}
t_{11}^{\mp \mp} & t_{12}^{\mp \mp} & \cdots \\
t_{21}^{\mp \mp} & t_{22}^{\mp \mp} & \cdots \\
\vdots & \vdots & \vdots
\end{array}\right)
$$

and

$$
\hat{r}^{ \pm \mp}=\left(\begin{array}{ccc}
r_{11}^{ \pm \mp} & r_{12}^{ \pm \mp} & \cdots \\
r_{21}^{ \pm \mp} & r_{22}^{ \pm \mp} & \cdots \\
\vdots & \vdots & \vdots
\end{array}\right)
$$

Here $t_{i j}^{++}\left(t_{i j}^{--}\right)$and $r_{i j}^{-+}\left(r_{i j}^{+-}\right)$denote the transmitted and reflected amplitudes in channel $i$ when there is a unit flux incident from the left (right) in channel $j$. Left to right- and right to left directions are denoted by + and - , respectively. The $S$-matrix expresses outgoing wave amplitudes in terms of ingoing ones on either side of the quasi-1D disordered wire via the scattering relations 11,21 


$$
\left(\begin{array}{c}
0 \\
0^{\prime}
\end{array}\right)=\widehat{S}\left(\begin{array}{c}
I \\
I^{\prime}
\end{array}\right)
$$

Here $I$ and $I^{\prime}\left(0\right.$ and $\left.0^{\prime}\right)$ denote ingoing (outgoing) amplitudes at the left and right sides of the disordered region, respectively. It follows from current conservation that e.g. for a unit flux which is incident from the right in channel $i$ one has

$$
\sum_{j=1}^{N}\left(\left|t_{j i}^{--}\right|^{2}+\left|r_{j i}^{-+}\right|^{2}\right)=1
$$

Likewise, one has also

$$
\sum_{j=1}^{N}\left(\left|t_{j i}^{++}\right|^{2}+\left|r_{j i}^{+-}\right|^{2}\right)=1
$$

Our task is now to derive miscroscopic realizations of the $S$-matrix in terms of the transfer matrices (30) and (32) describing transfer of Bloch waves across finite quasi-1D disordered systems. Let us remark that this may not always be possible as is seen here in the case of the three channel periodic model. Indeed, in this case we are able to identify proper transmission and reflection amplitudes obeying the symmetry relations (37) and (37a) only when assuming the random site energies on chains 1 and 2 to be identical,

$$
\varepsilon_{1 n}=\varepsilon_{2 n}, n=1,2, \ldots N_{L}
$$

rather than allowing the energies at all pairs of sites of the quasi-1D system to be uncorrelated, as in (27). In the absence of the correlation (38) the obtained transport amplitudes cannot be identified as actual reflection and transmission amplitudes of Bloch waves.

If the amplitude at the $n$th site in a channel $j$ corresponds to a Bloch wave $\psi_{n}^{j}=e^{i n k_{j}}$ (equation (19) ) then the $j, n$ and $j, n-1$ components of wave amplitude vectors, 


$$
\widehat{W}^{-1}\left(\begin{array}{c}
\vdots \\
\psi_{n}^{j} \\
\psi_{n-1}^{j} \\
\vdots
\end{array}\right)
$$

(with $\widehat{W} \equiv \widehat{V}_{0}$ or $\widehat{V}$ ), being transferred by the $n$-th slice have values $a e^{i n k j}$ and 0 , respectively; on the other hand if $\psi_{n}^{j}=e^{-i n k j}$ then the $j, n$ and $j, n-1$ components of the above vectors are 0 and $a^{\prime} e^{-i n k j}$. Hence, in accordance with our notation above for transmission and reflection matrix elements we denote the amplitudes at site $j, n$ and $j, n-1$ respectively as $a_{j, n-1}^{+}$and $a_{j, n-1}^{-}$since they correspond to amplitudes being transferred by the $n$-th slice and propagating in the $k_{j}$ and $-k_{j}$ directions, respectively. The transformed amplitude vectors

$$
\widehat{W}^{-1}\left(\begin{array}{c}
\vdots \\
\psi_{n}^{j} \\
\psi_{n-1}^{j} \\
\vdots
\end{array}\right)
$$

and

$$
\widehat{W}^{-1}\left(\begin{array}{c}
\vdots \\
\psi_{n+1}^{j} \\
\psi_{n}^{j} \\
\vdots
\end{array}\right)
$$

are thus rewritten, respectively, as 


$$
\widehat{W}^{-1}\left(\begin{array}{c}
\psi_{n}^{1} \\
\psi_{n-1}^{1} \\
\psi_{n}^{2} \\
\psi_{n-1}^{2} \\
\vdots
\end{array}\right) \equiv\left(\begin{array}{c}
a_{1, n-1}^{+} \\
a_{1, n-1}^{-} \\
a_{2, n-1}^{+} \\
a_{2, n-1}^{-} \\
\vdots
\end{array}\right) \text { and } \widehat{W}^{-1}\left(\begin{array}{c}
\psi_{n+1}^{1} \\
\psi_{n}^{1} \\
\psi_{n+1}^{2} \\
\psi_{n}^{2} \\
\vdots
\end{array}\right) \equiv\left(\begin{array}{c}
a_{1, n}^{+} \\
a_{1, n}^{-} \\
a_{2, n}^{+} \\
a_{2, n}^{-} \\
\vdots
\end{array}\right) \text {. }
$$

Using a similar notation for wave amplitudes transferred from $n=0$ to $n=N_{L}$ across a disordered wire of length $L=N_{L} a$, the wave transfer equations in the Bloch representation, obtained by iterating (12-13) read

$$
\left(\begin{array}{c}
a_{1, L}^{+} \\
a_{1, L}^{-} \\
a_{2, L}^{+} \\
a_{2, L}^{-}
\end{array}\right)=\widehat{X}_{0 L}\left(\begin{array}{c}
a_{1,0}^{+} \\
a_{1,0}^{-} \\
a_{2,0}^{+} \\
a_{2,0}^{-}
\end{array}\right),
$$

and

$$
\left(\begin{array}{c}
a_{1, L}^{+} \\
a_{1, L}^{-} \\
a_{2, L}^{+} \\
a_{2, L}^{-} \\
a_{3, L}^{+} \\
a_{3, L}^{-}
\end{array}\right)=\widehat{Y}_{L}\left(\begin{array}{c}
a_{1,0}^{+} \\
a_{1,0}^{-} \\
a_{2,0}^{+} \\
a_{2,0}^{-} \\
a_{3,0}^{+} \\
a_{3,0}^{-}
\end{array}\right),
$$

where $\widehat{X}_{0 L}$ is given in (30) and $\widehat{Y}_{L}$ stands for $\widehat{X}_{L}^{\prime}$ and $\widehat{X}^{\prime \prime}{ }_{L}$ in (32) with parameters defined by (18.a) and (24) and by (18.b) and (25), respectively.

In order to derive the $S$-matrices for our two- and three channel wire models we first rewrite (40-41) in the forms of equations involving outgoing amplitudes on the left side and 
incoming ones as the right side, as in (36). In the notation of (40) and (41) we have e.g. for $N=3$

$$
(I) \equiv\left(\begin{array}{c}
a_{1,0}^{+} \\
a_{2,0}^{+} \\
a_{3,0}^{+}
\end{array}\right),\left(I^{\prime}\right) \equiv\left(\begin{array}{c}
a_{1, L}^{-} \\
a_{2, L}^{-} \\
a_{3, L}^{-}
\end{array}\right),(O) \equiv\left(\begin{array}{c}
a_{1,0}^{-} \\
a_{2,0}^{-} \\
a_{3,0}^{-}
\end{array}\right),\left(O^{\prime}\right) \equiv\left(\begin{array}{c}
a_{1, L}^{+} \\
a_{2, L}^{+} \\
a_{3, L}^{+}
\end{array}\right)
$$

and so we rearrange (40-41) in the form

$$
\widehat{A}_{2,3}\left(\begin{array}{c}
O \\
O^{\prime}
\end{array}\right)=\widehat{B}_{2,3}\left(\begin{array}{c}
I \\
I^{\prime}
\end{array}\right),
$$

where the pairs of matrices $\widehat{A}_{2}$ and $\widehat{B}_{2}$ and $\widehat{A}_{3}$ and $\widehat{B}_{3}$ correspond to two- and three channel cases, respectively. Using the notation $\left(\widehat{X}_{0 L}\right)_{i j} \equiv X_{i j}$ and $\left(\widehat{Y}_{L}\right)_{i j} \equiv Y_{i j}$ for the matrix elements in (30) and (32), respectively, we find

$$
\begin{aligned}
& \widehat{A}_{2}=\left(\begin{array}{llll}
-X_{12} & -X_{14} & 1 & 0 \\
-X_{22} & -X_{24} & 0 & 0 \\
-X_{32} & -X_{34} & 0 & 1 \\
-X_{42} & -X_{44} & 0 & 0
\end{array}\right), \widehat{B}_{2}=\left(\begin{array}{cccc}
X_{11} & X_{13} & 0 & 0 \\
X_{21} & X_{23} & -1 & 0 \\
X_{31} & X_{33} & 0 & 0 \\
X_{41} & X_{43} & 0 & -1
\end{array}\right), \\
& \widehat{A}_{3}=\left(\begin{array}{cccccc}
-Y_{12} & -Y_{14} & -Y_{16} & 1 & 0 & 0 \\
-Y_{22} & -Y_{24} & -Y_{26} & 0 & 0 & 0 \\
-Y_{32} & -Y_{34} & -Y_{36} & 0 & 1 & 0 \\
-Y_{42} & -Y_{44} & -Y_{46} & 0 & 0 & 0 \\
-Y_{52} & -Y_{54} & -Y_{56} & 0 & 0 & 1 \\
-Y_{62} & -Y_{64} & -Y_{66} & 0 & 0 & 0
\end{array}\right), \widehat{B}_{3}=\left(\begin{array}{cccccc}
Y_{11} & Y_{13} & Y_{15} & 0 & 0 & 0 \\
Y_{21} & Y_{23} & Y_{25} & -1 & 0 & 0 \\
Y_{31} & Y_{33} & Y_{35} & 0 & 0 & 0 \\
Y_{41} & Y_{43} & Y_{45} & 0 & -1 & 0 \\
Y_{51} & Y_{53} & Y_{55} & 0 & 0 & 0 \\
Y_{61} & Y_{63} & Y_{65} & 0 & 0 & -1
\end{array}\right)
\end{aligned}
$$

The $S$-matrices for the two- and three channel cases are those given by $\widehat{S}=\widehat{A}_{2}^{-1} \widehat{B}_{2}$ and $\widehat{S}=\widehat{A}_{3}^{-1} \widehat{B}_{3}$, respectively. After inverting $\widehat{A}_{2}$ and $\widehat{A}_{3}$ we finally obtain, successively for the two- and three channel cases: 


$$
\widehat{S}=\frac{1}{\delta}\left(\begin{array}{cccc}
\delta_{1} & \delta_{2} & X_{44} & -X_{24} \\
\delta_{3} & \delta_{4} & -X_{42} & X_{22} \\
X_{11} \delta+X_{21} \delta_{5} & X_{13} \delta+X_{23} \delta_{5} & -\delta_{5} & -\delta_{6} \\
+X_{41} \delta_{6} & +X_{43} \delta_{6} & & \\
X_{31} \delta+X_{21} \delta_{7} & X_{33} \delta+X_{23} \delta_{7} & -\delta_{7} & -\delta_{8} \\
+X_{41} \delta_{8} & +X_{43} \delta_{8} & &
\end{array}\right)
$$

where

$$
\begin{gathered}
\delta=X_{22} X_{44}-X_{24} X_{42}, \delta_{1}=X_{24} X_{41}-X_{44} X_{21}, \delta_{2}=X_{24} X_{43}-X_{44} X_{23} \\
\delta_{3}=X_{42} X_{21}-X_{41} X_{22}, \delta_{4}=X_{42} X_{23}-X_{22} X_{43}, \delta_{5}=X_{42} X_{14}-X_{12} X_{44} \\
\delta_{6}=X_{12} X_{24}-X_{22} X_{14}, \delta_{7}=X_{42} X_{34}-X_{32} X_{44}, \delta_{8}=X_{32} X_{24}-X_{22} X_{34}
\end{gathered}
$$

are second order subdeterminants of $\widehat{X}_{0 L}$;

$$
\widehat{S}=\left(\begin{array}{ll}
\widehat{S}_{1} & \widehat{S}_{3} \\
\widehat{S}_{2} & \widehat{S}_{4}
\end{array}\right)
$$

where

$$
\begin{aligned}
& \widehat{S}_{1}=\frac{1}{\Delta} \\
& \left(\begin{array}{ccc}
-\beta_{1} Y_{21}-\beta_{4} Y_{41}-\beta_{7} Y_{61} & -\beta_{1} Y_{23}-\beta_{4} Y_{43}-\beta_{7} Y_{63}-\beta_{1} Y_{25}-\beta_{4} Y_{45}-\beta_{7} Y_{65} \\
\beta_{2} Y_{21}+\beta_{5} Y_{41}+\beta_{8} Y_{61} & \beta_{2} Y_{23}+\beta_{5} Y_{43}+\beta_{8} Y_{63} & \beta_{2} Y_{25}+\beta_{5} Y_{45}+\beta_{8} Y_{65} \\
-\beta_{3} Y_{21}-\beta_{6} Y_{41}-\beta_{9} Y_{61} & -\beta_{3} Y_{23}-\beta_{6} Y_{43}-\beta_{9} Y_{63} & -\beta_{3} Y_{25}-\beta_{6} Y_{45}-\beta_{9} Y_{65}
\end{array}\right),
\end{aligned}
$$

$$
\begin{aligned}
& \widehat{S}_{2}=\frac{1}{\Delta} \\
& \left(\begin{array}{ccc}
Y_{11} \Delta+Y_{21} \Delta_{1}+Y_{41} \Delta_{4}+Y_{61} \Delta_{7} & Y_{13} \Delta+Y_{23} \Delta_{1}+Y_{43} \Delta_{4}+Y_{63} \Delta_{7} & Y_{15} \Delta+Y_{25} \Delta_{1}+Y_{45} \Delta_{4}+Y_{65} \Delta_{7} \\
Y_{31} \Delta-Y_{21} \Delta_{2}-Y_{41} \Delta_{5}-Y_{61} \Delta_{8} & Y_{33} \Delta-Y_{23} \Delta_{2}-Y_{43} \Delta_{5}-Y_{63} \Delta_{8} & Y_{35} \Delta-Y_{25} \Delta_{2}-Y_{45} \Delta_{5}-Y_{65} \Delta_{8} \\
Y_{51} \Delta+Y_{21} \Delta_{3}+Y_{41} \Delta_{6}+Y_{61} \Delta_{9} & Y_{53} \Delta+Y_{23} \Delta_{3}+Y_{43} \Delta_{6}+Y_{63} \Delta_{9} & Y_{55} \Delta+Y_{25} \Delta_{3}+Y_{45} \Delta_{6}+Y_{65} \Delta_{9}
\end{array}\right)
\end{aligned}
$$




$$
\begin{aligned}
& \widehat{S}_{3}=\frac{1}{\Delta}\left(\begin{array}{ccc}
\beta_{1} & \beta_{4} & \beta_{7} \\
-\beta_{2} & -\beta_{5} & -\beta_{8} \\
\beta_{3} & \beta_{6} & \beta_{9}
\end{array}\right), \\
& \widehat{S}_{4}=\frac{1}{\Delta}\left(\begin{array}{ccc}
-\Delta_{1} & -\Delta_{4} & -\Delta_{7} \\
\Delta_{2} & \Delta_{5} & \Delta_{8} \\
-\Delta_{3} & -\Delta_{6} & -\Delta_{9}
\end{array}\right),
\end{aligned}
$$

which involve subdeterminants of second order of $\widehat{Y}_{L}$,

$$
\begin{aligned}
& \beta_{1}=Y_{46} Y_{64}-Y_{44} Y_{66}, \beta_{2}=Y_{62} Y_{46}-Y_{42} Y_{66}, \beta_{3}=Y_{62} Y_{44}-Y_{42} Y_{64} \\
& \beta_{4}=Y_{24} Y_{66}-Y_{64} Y_{26}, \beta_{5}=Y_{22} Y_{66}-Y_{62} Y_{26}, \beta_{6}=Y_{22} Y_{64}-Y_{62} Y_{24} \\
& \beta_{7}=Y_{44} Y_{26}-Y_{24} Y_{46}, \beta_{8}=Y_{42} Y_{26}-Y_{22} Y_{46}, \beta_{9}=Y_{24} Y_{42}-Y_{22} Y_{44},
\end{aligned}
$$

as well as third order subderterminants of $\widehat{A}_{3}$ which result from the minors of various elements,

$$
\begin{aligned}
& \Delta_{1}=\min \left(\widehat{A}_{3}\right)_{24}, \Delta_{2}=\min \left(\widehat{A}_{3}\right)_{25}, \\
& \Delta_{3}=\min \left(\widehat{A}_{3}\right)_{26}, \Delta_{4}=\min \left(\widehat{A}_{3}\right)_{44}, \Delta_{5}=\min \left(\widehat{A}_{3}\right)_{45} \\
& \Delta_{6}=\min \left(\widehat{A}_{3}\right)_{46}, \Delta_{7}=\min \left(\widehat{A}_{3}\right)_{64}, \Delta_{8}=\min \left(\widehat{A}_{3}\right)_{65} \\
& \Delta_{9}=\min \left(\widehat{A}_{3}\right)_{66}, \text { and } \Delta=\operatorname{det} \widehat{A}_{3}
\end{aligned}
$$

The partial matrices $\widehat{S}_{j}, j=1,2,3,4$ in (48) clearly correspond to reflection and transmission matrices in (33).

The matrices (46) and (48,48a-d) are the $S$-matrix expressions for the two- and three channel quasi-1D systems in terms of characteristic quantum channel wavenumbers and of the tight-binding quantities in the transfer matrices (30) and (32) for weak disorder. Identification of these expressions with 33 -35) yields the transmission- anf reflection matrices for these quasi-1D disordered models under the proviso that the symmetry relation (37-37a) are obeyed. 


\section{RESULTS AND DISCUSSION}

The results for the transmission- and reflection matrices of two- and three channel tightbinding wires are applied in this section for finding the averaged transmission and reflection coefficients associated with the various channels, for weak disorder. These results allow us, in particular, to explicitely check the symmetry property (37) in the two-channel case, as well as in the three-channel case with free boundary conditions. On the other hand in the three-channel case with periodic boundary conditions we show that (37) is obeyed if one restricts the disorder to a correlated site energy disorder with identical site energies (38) on chains 1 and 2 and independent random energies on chain 3.

The results for the averaged transmission coefficients are used for obtaining the length of exponential localization from (11) and (11). Our exact quantum expressions of localization lengths for weak disorder reduce to the well-known Thouless expression for a 1D chain in the limit of vanishing interchain coupling $(h \rightarrow 0)$. It is useful, before presenting our results, to briefly recall the derivation of Thouless' result from the transfer matrix approach [19]. For a single disordered chain of length $L$ the localization length is given by

$$
\frac{1}{L_{c}}=-\lim _{N_{L} \rightarrow \infty}\left(2 N_{l}\right)^{-1}\left\langle\ln |t|^{2}\right\rangle
$$

where $|t|^{2}=\left|t^{--}\right|^{2}=\left|t^{++}\right|^{2}$ is the transmission coefficient which is related to the twodimensional transfer matrices $\widehat{X}_{n}$ (in the Bloch wave representation) for thin sections enclosing the $n$th site by [19]

$$
\frac{1}{t^{--}}=\left(\prod_{n=1}^{N_{L}} \widehat{X}_{n}\right)_{22} .
$$

This relation follows by transforming the $S$-matrix for scattering states into a transfer matrix

whose 22 -element is $1 / t^{--}$. Expanding the transfer matrix for the whole chain, $\prod_{n=1}^{N_{L}} \widehat{X}_{n}$, to first order in the uncorrelated site energies and performing the average in (49), using (27), yields 


$$
\frac{1}{L_{c}} \equiv \frac{1}{\xi}=\frac{\varepsilon_{0}^{2}}{8 \sin ^{2} k}, E=2 \cos k
$$

which is Thouless' expression for the localization length in the tight-binding band, for weak gaussian disorder 13.

For convenience of the following discussion for two- and three channel systems, the explicit forms of the transmission and reflection coefficients obtained by identifying the $S$ matrix (33-35) successively with (46) and (48, 48.a-d) and replacing the transfer matrix

elements entering in these expressions by their explicit forms in (30-32) are given in the appendix.

\section{A. Two-channel wires}

By averaging the partial transmission- and reflection coefficients given by (A.1-A.4) over the disorder, using (22.a) and (27), we obtain successively

$$
\begin{gathered}
\left\langle\left|t_{11}^{--}\right|^{2}\right\rangle=1-\frac{N_{L} \varepsilon_{0}^{2}}{8}\left(\frac{1}{\sin ^{2} k_{1}}+\frac{2}{\sin k_{1} \sin k_{2}}\right), \\
\left\langle\left|t_{22}^{--}\right|^{2}\right\rangle=1-\frac{N_{L} \varepsilon_{0}^{2}}{8}\left(\frac{1}{\sin ^{2} k_{2}}+\frac{2}{\sin k_{1} \sin k_{2}}\right), \\
\left\langle\left|t_{12}^{--}\right|^{2}\right\rangle=\left\langle\left|t_{21}^{--}\right|^{2}\right\rangle=\frac{N_{L} \varepsilon_{0}^{2}}{8 \sin k_{1} \sin k_{2}}, \\
\left\langle\left|r_{11}^{-+}\right|^{2}\right\rangle=\frac{N_{L} \varepsilon_{0}^{2}}{8 \sin ^{2} k_{1}} \\
\left\langle\left|r_{22}^{-+}\right|^{2}\right\rangle=\frac{N_{L} \varepsilon_{0}^{2}}{8 \sin ^{2} k_{2}}
\end{gathered}
$$




$$
\left\langle\left|r_{12}^{-+}\right|^{2}\right\rangle=\left\langle\left|r_{21}^{-+}\right|^{2}\right\rangle \frac{N_{L} \varepsilon_{0}^{2}}{8 \sin k_{1} \sin k_{2}}
$$

The expressions (52-57) are consistent with the symmetry relations (37) resulting from current conservation.

The inverse localization length for weak disorder is obtained by expanding (11) to lowest order in the random site energies using (11) and (52-54). It is given by

$$
\frac{1}{L_{c}} \equiv \frac{1}{L_{0 c}}=\frac{\varepsilon_{0}^{2}}{32}\left(\frac{1}{\sin k_{1}}+\frac{1}{\sin k_{2}}\right)^{2},
$$

for energies $E$ restricted to the Bloch bands $E=h+2 \cos k_{1}$, and $E=-h+2 \cos k_{2}$. This exact expression for weak disorder reveals three important properties:

1. It proves miscoscopically that all states in the Bloch energy bands of two-channel quasi-1D disordered systems are localized.

2. In the absence of interchain hopping $(h=0)$ it reduces to the localization length (51) for a $1 \mathrm{D}$ chain described by the Anderson model. For weak interchain hopping (58) becomes

$$
\frac{1}{L_{0 c}}=\frac{\varepsilon_{0}^{2}}{8 \sin ^{2} k}\left[1+\frac{h^{2}}{4 \sin ^{2} k}\left(1+3 \cot ^{2} k\right)+O\left(h^{4}\right)\right],
$$

which is valid for energies sufficiently close to the band centre of the $h=0$ energy band, $E=2 \cos k$. This shows that a weak interchain hopping enhances localization in comparison to the purely $1 \mathrm{D}$ case, i.e. $L_{0 c}<\xi$.

3. For large interchain hopping rates, i.e. $|h|>>|E|$ (where $E$ is of the order of the fermi energy) we have $\sin k_{1,2} \simeq \sqrt{1-h^{2} / 4},|h| / 2 \leq 1$, which yields 


$$
\frac{1}{L_{c}} \simeq \frac{\varepsilon_{0}^{2}}{2} \frac{1}{4-h^{2}}=\frac{1}{\xi_{0}} \frac{4}{4-h^{2}}
$$

where $\xi_{0}=\varepsilon_{0}^{2} / 8$ is the $1 \mathrm{D}$ localization length (51) at the band centre. Thus at large hopping rates (with $|h|<2$ ) localization is also enhanced in comparison to 1D localization.

\section{B. Three-channel wires}

1. Free boundary conditions

The evaluation of the disorder averages of the transmission- and reflection coefficients in (A.5-A.6), using (27) and the explicit expressions in (A.7-A.9) with the tight-binding parameters (24) yields, to order $\varepsilon_{0}^{2}$,

$$
\begin{gathered}
\left\langle\left|t_{11}^{--}\right|^{2}\right\rangle=1-\frac{N_{L} \varepsilon_{0}^{2}}{32}\left(\frac{3}{\sin ^{2} k_{1}}+\frac{6}{\sin k_{1} \sin k_{3}}+\frac{4}{\sin k_{1} \sin k_{2}}\right) \\
\left\langle\left|t_{22}^{--}\right|^{2}\right\rangle=1-\frac{N_{L} \varepsilon_{0}^{2}}{32}\left(\frac{4}{\sin ^{2} k_{2}}+\frac{4}{\sin k_{2} \sin k_{3}}+\frac{4}{\sin k_{1} \sin k_{2}}\right) \\
\left\langle\left|t_{33}^{--}\right|^{2}\right\rangle=1-\frac{N_{L} \varepsilon_{0}^{2}}{32}\left(\frac{3}{\sin ^{2} k_{3}}+\frac{6}{\sin k_{1} \sin k_{3}}+\frac{4}{\sin k_{2} \sin k_{3}}\right) \\
\left\langle\left|t_{12}^{--}\right|^{2}\right\rangle=\left\langle\left|t_{21}^{--}\right|^{2}\right\rangle=\frac{2 N_{L} \varepsilon_{0}^{2}}{32 \sin k_{1} \sin k_{2}} \\
\left\langle\left|t_{13}^{--}\right|^{2}\right\rangle=\left\langle\left|t_{31}^{--}\right|^{2}\right\rangle=\frac{3 N_{L} \varepsilon_{0}^{2}}{32 \sin k_{1} \sin k_{3}}
\end{gathered}
$$




$$
\begin{gathered}
\left\langle\left|t_{23}^{--}\right|^{2}\right\rangle=\left\langle\left|t_{32}^{--}\right|^{2}\right\rangle=\frac{2 N_{L} \varepsilon_{0}^{2}}{32 \sin k_{2} \sin k_{3}} \\
\left\langle\left|r_{11}^{-+}\right|^{2}\right\rangle=\frac{3 N_{L} \varepsilon_{0}^{2}}{32 \sin ^{2} k_{1}}, \\
\left\langle\left|r_{22}^{-+}\right|^{2}\right\rangle=\frac{4 N_{L} \varepsilon_{0}^{2}}{32 \sin ^{2} k_{2}}, \\
\left\langle\left|r_{33}^{-+}\right|^{2}\right\rangle=\frac{3 N_{L} \varepsilon_{0}^{2}}{32 \sin ^{2} k_{3}}, \\
\left\langle\left|r_{13}^{-+}\right|^{2}\right\rangle=\left\langle\left|r_{31}^{-+}\right|^{2}\right\rangle \frac{3 N_{L} \varepsilon_{0}^{2}}{\sin _{12} k_{1} \sin k_{3}}
\end{gathered}
$$

Again, the expressions (61,72) obey the current conservation property (37) for the three channel case: the reduction of the intra-channel transmission coefficients due to scattering by the disordered wire is exactly compensated by the occurence of interchannel transmissions and by reflections.

For the inverse localization length we obtain, from (111),(1i) and (61--66), 
$\frac{1}{L_{c}}=\frac{\varepsilon_{0}^{2}}{64}\left(\frac{1}{\sin ^{2} k_{1}}+\frac{4}{3} \frac{1}{\sin ^{2} k_{2}}+\frac{1}{\sin ^{2} k_{3}}+\frac{4}{3 \sin k_{1} \sin k_{2}}+\frac{4}{3 \sin k_{2} \sin k_{3}}+\frac{2}{\sin k_{1} \sin k_{3}}\right)$

where $k_{1}, k_{2}, k_{3}$ are defined by (18.a). Like (58), this expression is exact to order $\varepsilon_{0}^{2}$ for weak disorder. It demonstrates that the eigenstates in the Bloch bands of the three channel quasi-1D system with free boundary conditions are localized. It too reduces to the Thouless result (51) in 1D for vanishing interchain hopping. For small values of $|h|$, in particular for $|h|<<1$ for $E \rightarrow 0$, (73) reduces to

$$
\frac{1}{L_{c}}=\frac{\varepsilon_{0}^{2}}{8 \sin ^{2} k_{2}}\left[1+\frac{h^{2}}{\sin ^{2} k_{2}}\left(1+\frac{1}{2} \cos ^{2} k_{2}+\frac{1}{4} \cot ^{2} k_{2}\right)+O\left(h^{4}\right)\right]
$$

and for large values $\left(h^{2}>>E^{2} / 2\right)$ restricted to $|h|<2$ it becomes

$$
\frac{1}{L_{c}} \simeq \frac{\varepsilon_{0}^{2}}{8} \frac{1}{2-h^{2}}
$$

which shows, in particular, that $L_{c}$ is increased with respect to the $1 \mathrm{D}$ value at the band centre $\left(\xi_{0}\right)$ for $|h|$ (in units of the interchain hopping rate) less than 1 . Thus, the domain of interchain hopping rates in which (73) leads to localization lengths larger than $\xi_{0}$ is defined by

$$
|E|<<\sqrt{2}|h|<\sqrt{2}
$$

\section{Periodic boundary conditions}

We first note that in this case the $S$-matrix constructed from the transfer matrix $\widehat{X}_{L}$ " in Sect. III is unitary (i.e. (37) and (37a) are verified) only in the case where (38) 
is obeyed i.e. when chains 1 and 2 have identical random site-energies while the random site energies on chain 3 are arbitrary. This may be readily seen by expressing the averages of the transmission- and reflection coefficients in (A.5) and (A.6) to second order in the site energies in terms of the averages of (A.7-A.9) assuming that site energies belonging to sites $m \neq n$ on the same chain or on different ones are uncorrelated i.e. $\left\langle\varepsilon_{i m} \varepsilon_{j n}\right\rangle=0$. In this way we find

$$
\begin{aligned}
\sum_{i, j=1}^{3}\left(\left\langle\left|t_{i j}^{--}\right|^{2}\right\rangle+\left\langle\left|r_{i j}^{-+}\right|^{2}\right\rangle\right)= & 3+2 N_{L}\left[\left(\left\langle c_{n}^{2}\right\rangle+\left\langle f_{n}^{2}\right\rangle-2\left\langle c_{n} f_{n}\right\rangle\right)\right. \\
& +\left(\left\langle d_{n}^{2}\right\rangle+\left\langle q_{n}^{2}\right\rangle-2\left\langle d_{n} q_{n}\right\rangle\right) \\
& \left.+\left(\left\langle g_{n}^{2}\right\rangle+\left\langle p_{n}^{2}\right\rangle-2\left\langle g_{n} p_{n}\right\rangle\right)\right] .
\end{aligned}
$$

Now, for this relation to be compatible with current conservation we require

$$
c_{n}=f_{n}, d_{n}=q_{n} \text { and } g_{n}=p_{n}
$$

and from the definition (25) it follows that the equalities (78) are fulfilled with (38) i.e. for identical site energies in the chains 1 and 2. Only under this condition does the $S$-matrix for the periodic 3 -channel system represent a true scattering matrix

The explicit second order expressions for the averaged transmission- and reflection coefficients obtained from (A.5-A.9) and (25), for random gaussian site energies (27) in the presence of the correlation (38) are

$$
\begin{gathered}
\left\langle\left|t_{11}^{--}\right|^{2}\right\rangle=1-\frac{N_{L} \varepsilon_{0}^{2}}{36}\left(\frac{5}{\sin ^{2} k_{1}}+\frac{8}{\sin k_{1} \sin k_{2}}\right), \\
\left\langle\left|t_{22}^{--}\right|^{2}\right\rangle=\left\langle\left|t_{33}^{--}\right|^{2}\right\rangle=1-\frac{N_{L} \varepsilon_{0}^{2}}{36}\left(\frac{5}{\sin ^{2} k_{2}}+\frac{4}{\sin ^{2} k_{2}}+\frac{4}{\sin k_{1} \sin k_{2}}\right),
\end{gathered}
$$




$$
\left\langle\left|t_{12}^{--}\right|^{2}\right\rangle=\left\langle\left|t_{21}^{--}\right|^{2}\right\rangle=\left\langle\left|t_{13}^{--}\right|^{2}\right\rangle=\left\langle\left|t_{31}^{--}\right|^{2}\right\rangle=\frac{2 N_{L} \varepsilon_{0}^{2}}{36} \frac{1}{\sin k_{1} \sin k_{2}}
$$

$$
\left\langle\left|t_{23}^{--}\right|^{2}\right\rangle=\left\langle\left|t_{32}^{--}\right|^{2}\right\rangle=\frac{2 N_{L} \varepsilon_{0}^{2}}{36} \frac{1}{\sin ^{2} k_{2}}
$$

$$
\left\langle\left|r_{11}^{-+}\right|^{2}\right\rangle=\frac{5 N_{L} \varepsilon_{0}^{2}}{36} \frac{1}{\sin ^{2} k_{1}}
$$

$$
\begin{gathered}
\left\langle\left|r_{22}^{-+}\right|^{2}\right\rangle=\left\langle\left|r_{33}^{-+}\right|^{2}\right\rangle=\frac{5 N_{L} \varepsilon_{0}^{2}}{36} \frac{1}{\sin ^{2} k_{2}} \\
\left\langle\left|r_{12}^{-+}\right|^{2}\right\rangle=\left\langle\left|r_{21}^{-+}\right|^{2}\right\rangle=\left\langle\left|r_{13}^{-+}\right|^{2}\right\rangle=\left\langle\left|r_{31}^{-+}\right|^{2}\right\rangle=\frac{N_{L} \varepsilon_{0}^{2}}{36} \frac{2}{\sin k_{1} \sin k_{2}} \\
\left\langle\left|r_{23}^{-+}\right|^{2}\right\rangle=\left\langle\left|r_{32}^{-+}\right|^{2}\right\rangle=\frac{2 N_{L} \varepsilon_{0}^{2}}{36} \frac{1}{\sin ^{2} k_{2}}
\end{gathered}
$$

The inverse localization length associated with the conductance (1) obtained from $(79-82)$ is

$$
\frac{1}{L_{c}}=\frac{\varepsilon_{0}^{2}}{216}\left(\frac{5}{\sin ^{2} k_{1}}+\frac{14}{\sin ^{2} k_{2}}+\frac{8}{\sin k_{1} \sin k_{2}}\right)
$$

where $k_{1}$ and $k_{2}$ are defined by $(18 \mathrm{~b})$. This expression which proves localization in periodic 3-channel systems, reduces again to the $1 \mathrm{D}$ result (51) for $h=0$. For small $h$ it is (with $E=2 \cos k$ )

$$
\frac{1}{L_{c}} \simeq \frac{\varepsilon_{0}^{2}}{8 \sin ^{2} k}\left[1+\frac{h^{2}}{2 \sin ^{2} k}\left(1+\frac{70}{27} \cot ^{2} k\right)\right]
$$


which shows enhanced localization for weak interchain hopping. On the other hand for $|h|>>|E|(86)$ becomes

$$
\frac{1}{L_{c}} \simeq \frac{5 \varepsilon_{0}^{2}}{216} \frac{1}{1-h^{2}}
$$

which leads to increased localization lengths, $L_{c}>\xi_{0}$, for $h^{2}<22 / 27$. Thus the domain where the localization length in the periodic 3-channel system is larger than the $1 \mathrm{D}$-value is defined by

$$
|E|<<|h|<0.906 \ldots
$$

Our exact microscopic results for two and three-channel systems indicate that in all case while in the three channel cases a weak interchain hopping decreases the localization length from its 1D-value. For strong interchain hopping a similar decrease persists in the two-channel case while in the three-channel cases the localization length increases with respect to the $1 \mathrm{D}$ result over restricted domains of hopping parameters defined by (75) and (89). This suggests the possible existence of quasi-metallic domains for the three-channel systems in some ranges of length scales lying between their localization lengths and the 1D localization length (of the order of the mean free path $\ell[10]$ ), in the considered ranges of large hopping. In contrast, we recall that for many-channel random wires $(N>>1)$ the metallic domain exists over a wide range of mesoscopic lengths defined by (5).

\section{APPENDIX}

For briefness' sake we only discuss explicit expressions for transmission amplitudes $t_{i j}^{--}$ and reflection amplitudes $r_{i j}^{-+}$relating to outgoing waves to the left of the disordered sample in the scattering equations (36) (see (33) and (42)). 


\section{Two-channel wires}

Identification of (33) and (46) yields

$$
\begin{aligned}
& \left|t_{11}^{--}\right|^{2}=\frac{\left|X_{44}\right|^{2}}{|\delta|^{2}},\left|t_{12}^{--}\right|^{2}=\frac{\left|X_{24}\right|^{2}}{|\delta|^{2}}, \\
& \left|t_{21}^{--}\right|^{2}=\frac{\left|X_{42}\right|^{2}}{|\delta|^{2}} \text { and }\left|t_{22}^{--}\right|^{2}=\frac{\left|X_{22}\right|^{2}}{|\delta|^{2}},
\end{aligned}
$$

and, to lowest order in the random site energies, using (47) and (30),

$$
\begin{aligned}
& \left|r_{11}^{-+}\right|^{2}=\left|X_{21}\right|^{2},\left|r_{12}^{-+}\right|^{2}=\left|X_{23}\right|^{2}, \\
& \left|r_{21}^{-+}\right|^{2}=\left|X_{41}\right|^{2},\left|r_{22}^{-+}\right|^{2}=\left|X_{43}\right|^{2}
\end{aligned}
$$

¿From the definition of $\delta$ in (47) and the explicit form of the elements $X_{i j}$ in (30-31), we obtain

$$
\begin{aligned}
|\delta|^{2} & =1+\sum_{m, n=1}^{N_{L}}\left[a_{1 m} a_{1 n}+a_{2 m} a_{2 n}+2 b_{m} b_{n} \cos (m-n)\left(k_{1}-k_{2}\right)\right] \\
\left|X_{22}\right|^{2}= & +\sum_{m, n} a_{1 m} a_{1 n},\left|X_{44}\right|^{2}=1+\sum_{m, n} a_{2 m} a_{2 n} \\
\left|X_{12}\right|^{2} & =\sum_{m, n} a_{1 m} a_{2 n} \cos 2(m-n) k_{1},\left|X_{24}\right|^{2}=\sum_{m, n} b_{m} b_{n} \cos (m-n)\left(k_{1}-k_{2}\right) \\
\left|r_{11}^{-+}\right|^{2} & =\sum_{m, n} a_{1 m} a_{1 n} \cos 2(m-n) k_{1} \\
\left|r_{12}^{-+}\right|^{2} & =\sum_{m, n} b_{m} b_{n} \cos (m-n)\left(k_{1}+k_{2}\right) \\
\left|r_{21}^{-+}\right|^{2} & =\sum_{m, n} b_{m} b_{n} \cos (m-n)\left(k_{1}+k_{2}\right) \\
\left|r_{22}^{-+}\right|^{2} & =\sum_{m, n} a_{2 m} a_{2 n} \cos 2(m-n) k_{2}
\end{aligned}
$$

2. Three channel wires

The identification of (48.c) with the transmission matrix $\hat{t}^{--}$in (34) yields 


$$
\begin{aligned}
& \left|t_{11}^{--}\right|^{2}=\frac{\left|\beta_{1}\right|^{2}}{|\Delta|^{2}},\left|t_{12}^{--}\right|^{2}=\frac{\left|\beta_{4}\right|^{2}}{|\Delta|^{2}},\left|t_{13}^{--}\right|^{2}=\frac{\left|\beta_{7}\right|^{2}}{|\Delta|^{2}}, \\
& \left|t_{21}^{--}\right|^{2}=\frac{\left|\beta_{2}\right|^{2}}{|\Delta|^{2}},\left|t_{22}^{--}\right|^{2}=\frac{\left|\beta_{5}\right|^{2}}{|\Delta|^{2}},\left|t_{23}^{--}\right|^{2}=\frac{\left|\beta_{8}\right|^{2}}{|\Delta|^{2}}, \\
& \left|t_{31}^{--}\right|^{2}=\frac{\left|\beta_{3}\right|^{2}}{|\Delta|^{2}},\left|t_{32}^{--}\right|^{2}=\frac{\left|\beta_{6}\right|^{2}}{|\Delta|^{2}},\left|t_{33}^{--}\right|^{2}=\frac{\left|\beta_{9}\right|^{2}}{|\Delta|^{2}} .
\end{aligned}
$$

Similarly, the reflection amplitudes $r_{i j}^{-+}$are obtained by identifying $\widehat{S}_{1}$, in (48.a) with (35). For analyzing the reflection coefficients for weak disorder it suffices to find the amplitudes to linear order in the site energies. Such linear contributions are associated with $\beta_{j}$-terms having a zeroth order contribution (of modulus one). From (48.a) and (48.e) we thus obtain

$$
\begin{aligned}
& \left|r_{11}^{-+}\right|^{2}=\left|Y_{21}\right|^{2},\left|r_{12}^{-+}\right|^{2}=\left|Y_{23}\right|^{2},\left|r_{13}^{-+}\right|^{2}=\left|Y_{25}\right|^{2}, \\
& \left|r_{21}^{-+}\right|^{2}=\left|Y_{41}\right|^{2},\left|r_{22}^{-+}\right|^{2}=\left|Y_{43}\right|^{2},\left|r_{23}^{-+}\right|^{2}=\left|Y_{45}\right|^{2}, \\
& \left|r_{31}^{-+}\right|^{2}=\left|Y_{61}\right|^{2},\left|r_{32}^{-+}\right|^{2}=\left|Y_{63}\right|^{2},\left|r_{33}^{-+}\right|^{2}=\left|Y_{65}\right|^{2}
\end{aligned}
$$

The explicit expressions of the $\left|\beta_{j}\right|^{2}$ and $|\Delta|^{2}$ in (A.5) (defined in (48.e,f) and of the reflection coefficients (A.6) in terms of the transfer matrix elements in (32) are given by

$$
\begin{aligned}
\left|\beta_{1}\right|^{2} & =1+\sum_{m, n}\left[a_{3 m} a_{3 n}+b_{2 m} b_{2 n}+2 d_{m} q_{n} \cos (m-n)\left(k_{3}-k_{2}\right)\right] \\
\left|\beta_{5}\right|^{2} & =1+\sum_{m, n}\left[a_{1 m} a_{1 n}+a_{3 m} a_{3 n}+2 g_{m} p_{n} \cos (m-n)\left(k_{3}-k_{1}\right)\right] \\
\left|\beta_{9}\right|^{2} & =1+\sum_{m, n}\left[a_{1 m} a_{1 n}+b_{2 m} b_{2 n}+2 c_{m} f_{n} \cos (m-n)\left(k_{2}-k_{1}\right)\right] \\
\left|\beta_{2}\right|^{2}= & \sum_{m, n} f_{m} f_{n} \cos (m-n)\left(k_{1}-k_{2}\right),\left|\beta_{4}\right|^{2}=\sum_{m} c_{m} c_{n} \cos (m-n)\left(k_{1}-k_{2}\right) \\
\left|\beta_{3}\right|^{2}= & \sum_{m, n} p_{m} p_{n} \cos (m-n)\left(k_{1}-k_{3}\right),\left|\beta_{7}\right|^{2}=\sum_{m, n} g_{m} g_{n} \cos (m-n)\left(k_{1}-k_{3}\right) \\
\left|\beta_{6}\right|^{2}= & \sum_{m, n} q_{m} q_{n} \cos (m-n)\left(k_{2}-k_{3}\right),\left|\beta_{8}\right|^{2}=\sum_{m, n} d_{m} d_{n} \cos (m-n)\left(k_{2}-k_{3}\right) \\
|\Delta|^{2}= & 1+\sum_{m, n}\left[a_{1 m} a_{1 n}+a_{3 m} a_{3 n}+b_{2 m} b_{2 n}+2 g_{m} p_{n} \cos (m-n)\left(k_{3}-k_{4}\right)\right. \\
& \left.+2 d_{m} q_{n} \cos (m-n)\left(k_{3}-k_{2}\right)+2 c_{m} f_{n} \cos (m-n)\left(k_{2}-k_{1}\right)\right]
\end{aligned}
$$




$$
\begin{aligned}
\left|r_{11}^{-+}\right|^{2} & =\sum_{m, n} a_{1 m} a_{1 n},\left|r_{22}^{-+}\right|^{2}=\sum_{m, n} b_{2 m} b_{2 n} \cos 2(m-n) k_{2}, \\
\left|r_{33}^{-+}\right|^{2} & =\sum_{m, n} a_{3 m} a_{3 n} \cos 2(m-n) k_{3},\left|r_{12}^{-+}\right|^{2}=\sum_{m, n} c_{m} c_{n} \cos (m-n)\left(k_{1}+k_{2}\right), \\
\left|r_{13}^{-+}\right|^{2} & =\sum_{m, n} g_{m} g_{n} \cos (m-n)\left(k_{1}+k_{3}\right),\left|r_{21}^{-+}\right|^{2}=\sum_{m, n} f_{m} f_{n} \cos (m-n)\left(k_{1}+k_{2}\right), \\
\left|r_{23}^{-+}\right|^{2} & =\sum_{m, n} d_{m} d_{n} \cos (m-n)\left(k_{2}+k_{3}\right),\left|r_{31}^{-+}\right|^{2}=\sum_{m, n} p_{m} p_{n} \cos (m-n)\left(k_{1}+k_{3}\right) \\
\left|r_{32}^{-+}\right|^{2} & =\sum_{m, n} q_{m} q_{n} \cos (m-n)\left(k_{2}+k_{3}\right),
\end{aligned}
$$

where the double summations run from $m=1$ to $m=N_{L}$ and $n=1$ to $n=N_{L}$. The site-dependent tight-binding parameters $a_{1 j}, a_{3 j}, b_{2 j}, c_{j}, d_{j}, f_{j}, g_{j}, p_{j}, q_{j}$ in the above expressions are defined by (24) for free boundary conditions and by (25) for periodic boundary conditions. Likewise the wavenumbers $k_{1}, k_{2}, k_{3}$ are given by (18.a) and by (18.b) for free- and for periodic boundary conditions, respectively. 


\section{REFERENCES}

[1] Y. Imry and R. Landauer, Rev. Mod. Phys. 71, 306 (1999).

[2] S. Datta, Electronic transport in Mesoscopic Systems (Cambridge University Press, 1995).

[3] B.J. van Wees et al., Phys. Rev. Letters 60, 848 (1988); D.A. Wharam et al., J. Phys. C: Solid State Phys. 21, 209 (1988); see also H. Van Houten and C.W.J. Beenakker, Phys. Today 49, No 7, 22 (1996).

[4] For recent reviews see A.D. Stone, P.A. Mello, K.A. Muttalib and J.L. Pichard in Mesoscopic Phenomena in Solids ed. by B.L. Altshuler, P.A. Lee and R.A. Webb (North Holland, Amsterdam, 1991); C.W.J. Beenakker, Rev. Mod. Phys. 69, 760 (1997).

[5] D.J. Thouless, Phys. Rev. Letters 39, 1167 (1977).

[6] Y. Imry, Introduction to Mesoscopic Physics (Oxford University, London 1995).

[7] Y. Imry, Europhys. Lett. 1, 249 (1986).

[8] O.N. Dorokhov, Zh. Eksp. Teor. Fiz. 85, 1040 (1983) [Sov. Phys. JETP 58, 606].

[9] P.W. Anderson, D.J. Thouless, E. Abrahams, and D.S. Fisher, Phys. Rev. B 22, 3519 (1980).

[10] D.J. Thouless, J. Phys. C: Solid State Phys. 6, 249 (1973).

[11] See e.g. M. Janssen, Phys. Rep. 295, 1 (1998).

[12] O.N. Dorokhov, Solid State Com. 44, 915 (1982).

[13] D.J. Thouless, in Ill Condensed Matter, edited by R. Balian, R. Maynard, and G. Toulouse (North Holland, Amsterdam, 1979).

[14] See e.g. D Mailly, C. Chapelier, and A. Benoit, Phys. Rev. Lett. 70, 2020 (1993).

[15] R. Johnston and H. Kunz, J. Phys. C: Solid State Phys. 16, 3895 (1983). 
[16] V.I. Oseledec, Trans. Moscow Math. Soc. 19, 197 (1968).

[17] V.N. Tutubalin, Theor. Prob. Appl. 13, 65 (1968); A.D. Vister, ibid. 15, 667 (1970).

[18] See also the reviews on localization in A. Crisanti, G. Paladin, A. Vulpiani, Products of Random Matrices (Springer, Berlin, 1993); M. Janssen, O. Viehweger, V. Fastenrath, and J. Hajdu, Introduction to the Theory of the Integer Quantum Hall Effect (VCH, New York, 1994).

[19] J.B. Pendry, Adv. Phys. 43, 461 (1994).

[20] P. Erdös and R.C. Herndon, Adv. Phys. 31, 65 (1982).

[21] P.W. Anderson and P.A. Lee, Supp. Prog. Theor. Phys. 69, 212 (1980). 